\title{
Collagen Crosslinking-assisted Treatment of a Bleb Leak: Enhancement of Vascularization around the Bleb
}

\author{
Zeynep Aktas ${ }^{1}$, Yavuz K Aribas ${ }^{2}$, Kamil Bilgihan ${ }^{3}$, Atike B Tefon ${ }^{4}$
}

\begin{abstract}
Aim and objective: To present a case with bleb leakage treated with collagen crosslinking (CXL).

Background: Bleb-related complications can cause serious problems even a long time after trabeculectomy. In this case report, we present a relatively unknown treatment method for bleb leakage which might be one of the long-term complications of trabeculectomy.

Case description: A 60-year-old male patient was admitted to our clinic with a decrease in his left vision. The patient had a history of left trabeculectomy. The bleb leakage was observed. Autologous blood was injected into the bleb area as the first line of treatment. Collagen crosslinking was applied to the bleb area upon the recurrence of the leakage. In the 1st week after the CXL, CXL was repeated in the bleb area upon the observation that the leakage recurred. It was observed that the leakage did not recur and bleb vascularization was triggered after CXL. Conclusion: Conjunctival CXL might be a non-invasive, viable method in the management of bleb leakage. It might be applied in conjunction with bandage contact lenses for the treatment of late bleb leaks as an alternative treatment method before going into surgical intervention. Clinical significance: As a result of conjunctival CXL application, collagen permeability reduction and neovascularization secondary to CXL application might be effective in preventing bleb leakage. However, further studies are needed to prove this.

Keywords: Bleb leaks, Collagen crosslinking, Trabeculectomy.

Journal of Current Glaucoma Practice (2021): 10.5005/jp-journals-10078-1290
\end{abstract}

\section{INTRODUCTION}

Trabeculectomy has been one of the most commonly performed glaucoma surgeries for years worldwide. However, bleb-related complications are not uncommon. In the tube vs trabeculectomy study, bleb leak occurred in $11 \%$ of cases in 5 years. ${ }^{1}$ Bleb leaks can be classified as early or late leaks. In cases with early bleb leak occurring 2 weeks after the surgery, leaks are usually secondary to inadequate conjunctival wound closure, cheese-wiring, or conjunctival buttonhole. ${ }^{2}$ However, a late-onset bleb leakage that occurs more than two weeks after trabeculectomy is not a rare condition mostly due to the use of antifibrotic agents causing ischemic, avascular, thin blebs. ${ }^{3}$

Many conventional methods are used to prevent bleb leakages such as autologous blood injections, fibrin, and cyanoacrylate glue applications. ${ }^{4}$ However, further complications may be seen after these interventions. Autologous blood injection is known to affect by increasing cellular proliferation in the bleb region and causing mechanical block by clot formation in leakage area. ${ }^{5}$ Hyphema in the anterior chamber and infection associated with autologous blood injection caused by autologous blood injections are known complications of autologous blood injection. ${ }^{6}$ Recurrent leakage may be seen after clot dissolution. There are publications related to the use of fibrin and cyanoacrylate, but the results are debatable., ${ }^{7,8}$

Bleb excision and conjunctival advancement are considered among the surgical treatments of late bleb leaks, although surgical intervention might be difficult due to the fragile conjunctiva in these patients. ${ }^{4}$

Collagen crosslinking $(C X L)$ is a method of inducing the formation of covalent bonds between collagen in corneal ectatic diseases. ${ }^{9}$ It is also shown to be beneficial in corneal edema and infectious conditions. ${ }^{10}$

\footnotetext{
1,3 Department of Ophthalmology, Gazi University Faculty of Medicine, Ankara, Turkey

${ }^{2,4}$ Department of Ophthalmology, Gaziantep Abdulkadir Yuksel State Hospital, Gaziantep, Turkey
}

Corresponding Author: Zeynep Aktas, Department of Ophthalmology, Gazi University Faculty of Medicine, Ankara, Turkey, Phone: +903122026327, e-mail: drzeynep2000@yahoo.com

How to cite this article: Aktas Z, Aribas YK, Bilgihan K, et al. Collagen Crosslinking-assisted Treatment of a Bleb Leak: Enhancement of Vascularization around the Bleb. J Curr Glaucoma Pract 2021;15(1):36-39.

Source of support: Nil

Conflict of interest: None

It has been shown that UV-A crosslinking treatment applied to bleb leakage area was found to be successful by Wang and Harasymowycz and Choy et al. ${ }^{11,12}$ In this report, we present a case of bleb leakage which treated with the conjunctival crosslinking application and increased vascularization around the bleb.

\section{Case Description}

The written consent to publish personal information and case details have been obtained from the patient. A 60-year-old male patient presented with decreased visual acuity in the left eye. The patient's medical history revealed that he underwent glaucoma surgery (trabeculectomy) on the left eye 10 years ago in another center. The intraocular pressure (IOP) was measured as $3 \mathrm{~mm} \mathrm{Hg}$. On anterior segment examination, Descemet membrane wrinkles, irregular pupil, shallow anterior chamber, and bleb leakage were noted (Fig. 1). On fundus examination, the patient was found to have 


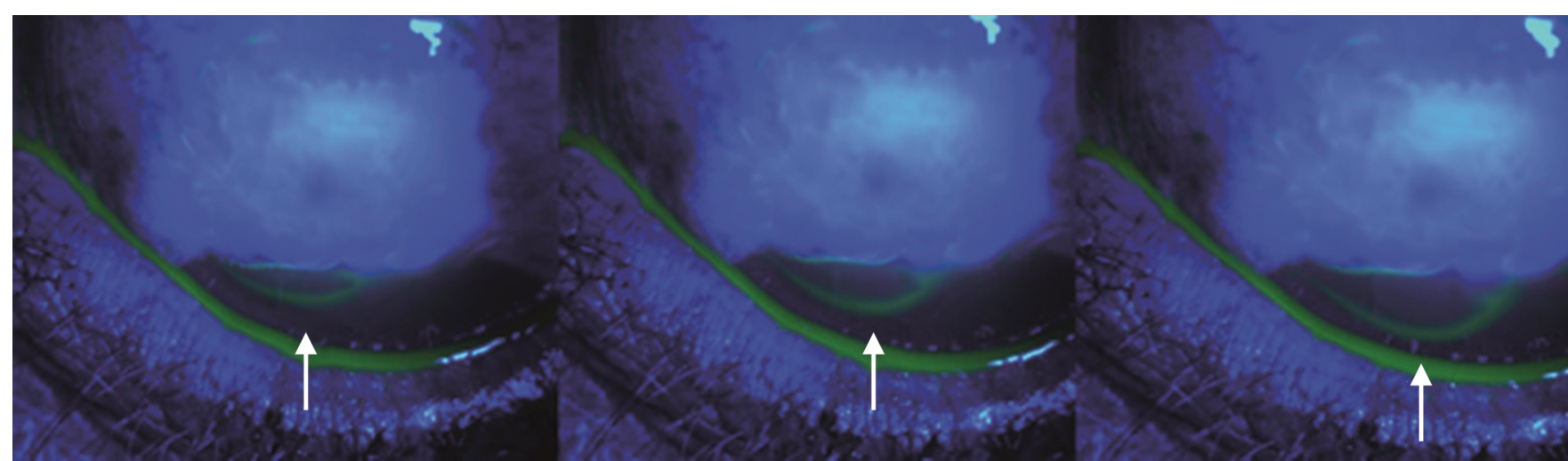

Fig. 1: Leakage from the bleb area is shown with the arrows at the baseline examination

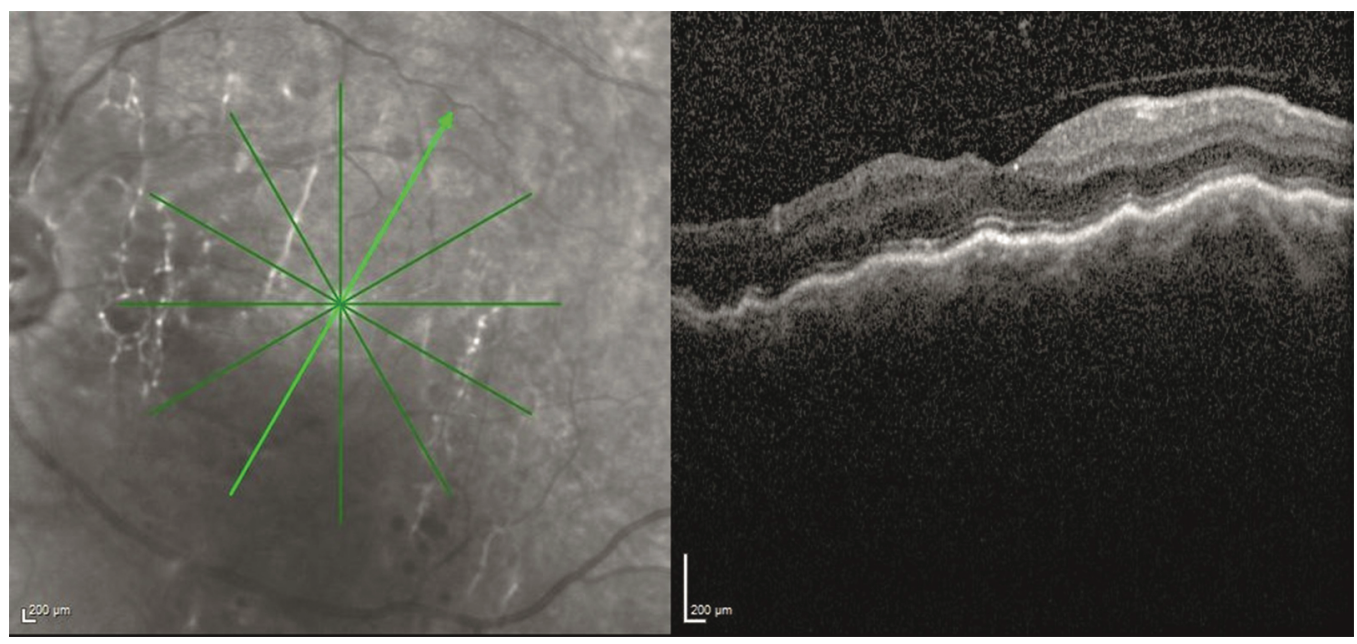

Fig. 2: Chorioretinal folds which is the sign of hypotony was seen on fundus optical coherence tomography (OCT) at the baseline examination

chorioretinal folds (Fig. 2). Autologous blood injection into the bleb region was performed as a first-line treatment. Leakage stopped just for 3 days after autologous blood injection but recurred after clot's dissolution on the bleb. CXL was considered after autologous blood injection was failed to stop leakage. UV-A was applied to the bleb site after the application of the riboflavin solution for 10 minutes. Hypertonic riboflavin solution was used during the crosslinking process to increase the conjunctival riboflavin penetration, while the cornea was being preserved by a cotton sponge. A $16 \times 16$ $\mathrm{mm}$ silicone hydrogel special custom-designed contact lens was used to stop the leakage until the therapeutic effect of the CXL has begun. When the contact lens was removed on the 3rd day, it was observed that the leak was ceased significantly. CXL was repeated a week after the procedure since the bleb was still leaking even decreased. However, one of the most striking findings was the vascularization around the bleb, which was not present at the first visit (Fig. 3). A month after the second CXL application, the leakage of the bleb stopped (Fig. 4A) and the IOP elevated to $14 \mathrm{~mm} \mathrm{Hg}$, although chorioretinal folds remained on the fundus examination. Vascularization around the bleb seemed to increase compared to that of the one we noticed on the first visit. Finally, at the 1st year follow-up, IOP was 12 and there was no bleb leakage (Fig. 4B).

\section{Discussion}

Trabeculectomy is still the most common glaucoma surgery performed by glaucoma specialists worldwide but it has many complications. ${ }^{4}$ One of these complications is bleb leakage which may be in the early and late postoperative period. ${ }^{4}$ Early leakage is often associated with poor surgical technique and inadequate wound healing. ${ }^{4}$ However, late-type leakage is caused by local changes in the bleb area, which is often caused by the antimetabolites. $^{3}$

In a recent study by Choy et al., CXL was shown to be successful in stopping the bleb leak in five patients, at different times, ranging from 1st to 4th weeks after the CXL application. ${ }^{12}$ In our case, the use of a bandage scleral contact lens might have contributed to the fast cessation of leakage after initial CXL treatment. There was no patient with hypotony-related complications in the study by Choy et al. ${ }^{12}$ However, in our case, hypotony maculopathy was developed and maculopathy was not responded to treatment of hypotony which might be due to a longer period of leakage.

In another study by Chan et al., ${ }^{13} \mathrm{CXL}$ was shown to decrease the permeability of conjunctiva and increase resistance to rupture in the cattle conjunctiva. ${ }^{13}$ Furthermore, CXL was shown to increase amid bands in the conjunctiva, which leads to strengthened conjunctival microstructure. It may be thought that the conjunctival CXL may be effective in preventing leaks because it strengthens the conjunctiva and reduces permeability. Also, it might be due to CXL-induced fibrovascular proliferation triggering vascularization at the bleb margins. In a recent study by Zhu et al., it was observed that CXL inhibited angiogenesis and lymphangiogenesis for the first 7 days at the corneal vascularization area, but later on, CXL enhanced the 

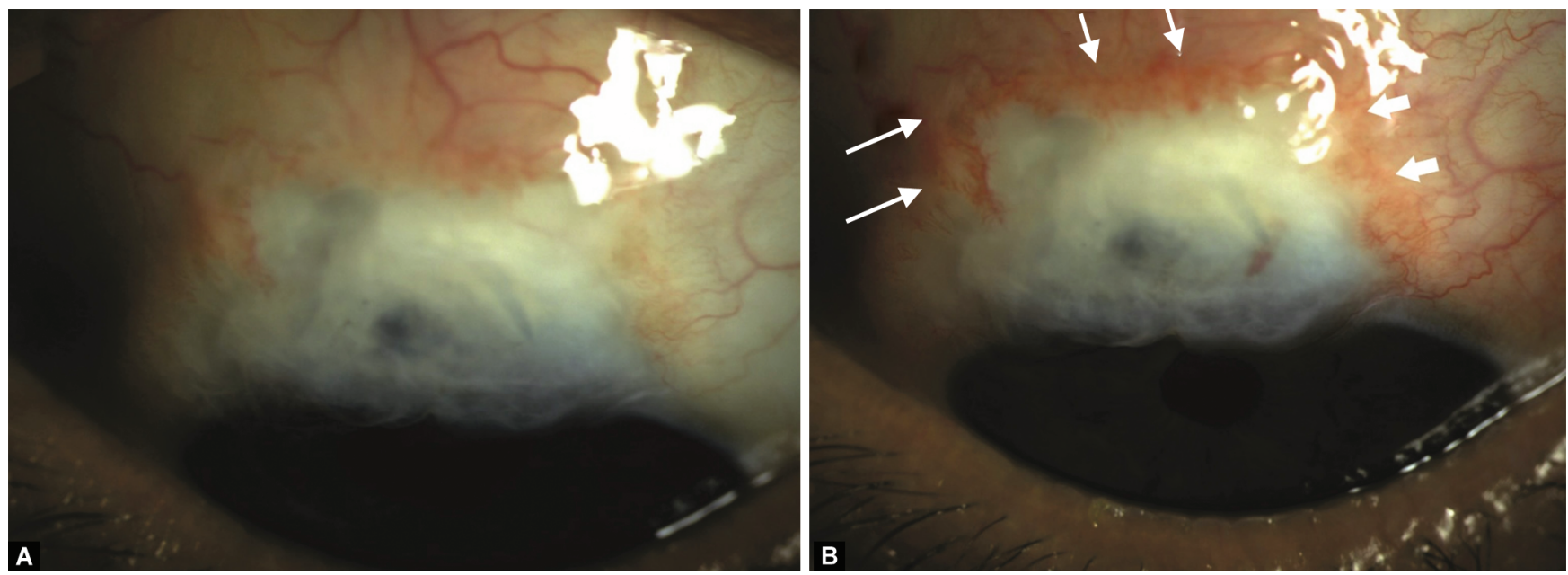

Figs $3 \mathrm{~A}$ and B: (A) Bleb area was seen before the CXL application at the baseline; (B) Vascularization at the bleb margin was seen 1 month after the 2nd CXL application. Vascularization was shown with the white arrows
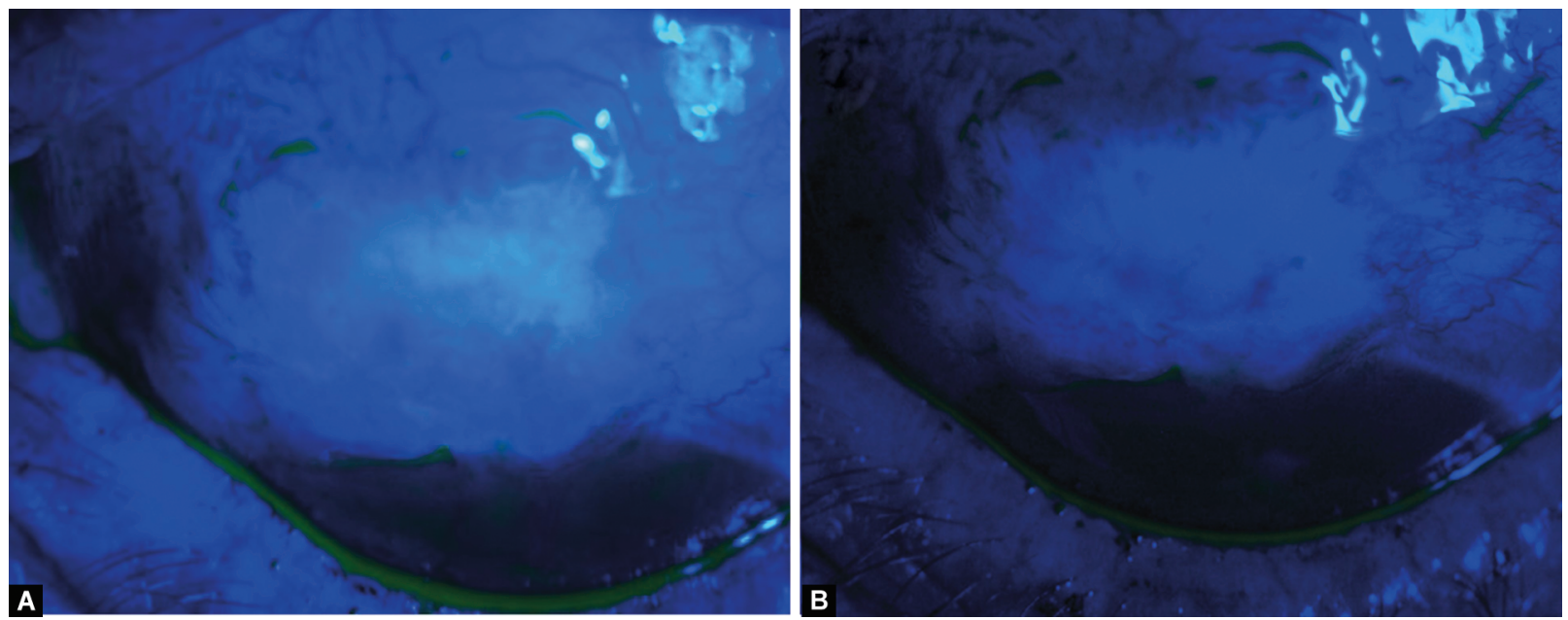

Figs 4A and B: (A) No leakage at the bleb area was seen 1 month after the 2 nd CXL application; (B) No leakage at the bleb area was detected 1 year after the 2 nd CXL application

effect of angiogenesis and lymphangiogenesis factors. ${ }^{14}$ In the same study, it has also been shown that inflammatory cytokines are similarly increased in CXL performed corneas after a temporary suppression period. ${ }^{14}$ Similarly, vascularization at the bleb border in our patient might be caused by a combination of CXL-induced proinflammatory cytokines and angiogenic factors.

In the present case, since we did not expect an acute cessation of the leak right after the $C X L$, we had to apply a scleral contact lens after the procedure. One might hypothesize that this might be the result of the scleral contact lens application. However, this should have been done for the patient's safety right after the CXL application. Furthermore, increased vascularization around the bleb right after the CXL application was significant and this finding made us think that increased inflammatory response induced by $C X L$ as reported previously, might have contributed to the healing of the bleb leak or at least shorten the healing time.

Therefore, conjunctival CXL might be a non-invasive, viable method for the management of bleb leakage. It might be applied in conjunction with bandage contact lenses for the treatment of late bleb leaks as an alternative treatment method before going into surgical intervention. Prospective case series are still needed to shed light on this subject.

\section{References}

1. Gedde SJ, Singh K, Schiffman JC, et al. Tube versus trabeculectomy study $\mathrm{G}$. The tube versus trabeculectomy study: interpretation of results and application to clinical practice. Curr Opin Ophthalmol 2012;23(2):118-126. DOI: 10.1097/ICU.0b013e32834ff2d1.

2. Alwitry A, Rotchford A, Patel V, et al. Early bleb leak after trabeculectomy and prognosis for bleb failure. Eye (Lond) 2009;23(4):858-863. DOI: 10.1038/eye.2008.130.

3. Bochmann F, Azuara-Blanco A. Interventions for late trabeculectomy bleb leak. Cochrane Database Syst Rev 2012;9(9):CD006769. DOI: 10.1002/14651858.CD006769.pub2.

4. Leung DY, Tham CC. Management of bleb complications after trabeculectomy. Semin Ophthalmol 2013;28(3):144-156. DOI: 10.3109/08820538.2013.771199.

5. Smith MF, Magauran R, Doyle JW. Treatment of postfiltration bleb leak by bleb injection of autologous blood. Ophthal Surg Lasers 1994;25(9):636-637. DOI: 10.3928/1542-8877-19940901-16.

6. Flynn WJ, Rosen WJ, Campbell DG. Delayed hyphema and intravitreal blood following intrableb autologous blood injection after 
trabeculectomy. Am J Ophthalmol 1997;124(1):115-116. DOI: 10.1016/ s0002-9394(14)71658-7.

7. Zalta $\mathrm{AH}$, Wieder $\mathrm{RH}$. Closure of leaking filtering blebs with cyanoacrylate tissue adhesive. Br J Ophthalmol 1991;75(3):170-173. DOI: 10.1136/bjo.75.3.170.

8. Asrani SG, Wilensky JT. Management of bleb leaks after glaucoma filtering surgery - use of autologous fibrin tissue glue as an alternative. Ophthalmology 1996;103(2):294-298. DOI: 10.1016/s01616420(96)30701-x.

9. Raiskup F, Spoerl E. Corneal crosslinking with riboflavin and ultraviolet A. I. Principles. Ocul Surf 2013;11(2):65-74. DOI: 10.1016/j. jtos.2013.01.002.

10. Bettis DI, Hsu M, Moshirfar M. Corneal collagen cross-linking for nonectatic disorders: a systematic review. J Refract Surg 2012;28(11):798-807. DOI: 10.3928/1081597X-20121011-09.
11. Wang Q, Harasymowycz P. Collagen cross-linking for late-onset bleb leakage: 1-year results. J Glaucoma 2016;25(3):E273-E276. DOI: 10.1097/IJG.0000000000000295.

12. Choy BNK, Zhu MM, Shum JWH, et al. Collagen crosslinking in the management of leaking cystic blebs: a prospective study. Graefes Arch Clin Exp Ophthalmol 2016;254(3):529-533. DOI: 10.1007/s00417015-3245-2.

13. Chan HMH, Choy BNK, Lai JSM. Effects of riboflavin and ultraviolet illumination on the biomechanical properties of conjunctiva. Ophthal Res 2018;60(2):87-93. DOI: 10.1159/000478051.

14. Zhu Y, Li L, Reinach PS, et al. Corneal collagen cross-linking with riboflavin and UVA regulates hemangiogenesis and lymphangiogenesis in rats. Invest Ophthalmol Vis Sci 2018;59(8): 3702-3712. DOI: 10.1167/iovs.17-23036. 